\title{
AKAP signaling complexes at the cytoskeleton
}

\author{
Dario Diviani and John D. Scott* \\ Howard Hughes Medical Institute and Vollum Institute, Oregon Health Sciences University, 3181 S. W. Sam Jackson Park Road, Portland, Oregon, \\ 97201, USA \\ *Author for correspondence (e-mail: scott@ohsu.edu) \\ Journal of Cell Science 114, 1431-1437 @ The Company of Biologists Ltd
}

\begin{abstract}
Summary
Targeting of protein kinases and phosphatases to the cytoskeleton enhances the regulation of signal transduction events. The assembly of cytoskeletal signaling complexes facilitates the relay of messages from membrane receptors to specific sites on the actin cytoskeleton. These signals influence fundamental cell properties, such as shape, movement and division. Targeting of the cAMP-dependent kinase (PKA) to the cytoskeleton is achieved through
\end{abstract}

interaction with $\underline{A}$-kinase anchoring proteins (AKAPs). AKAPs maintain multivalent signaling complexes by binding additional enzymes, including kinases and phosphatases.

Key words: Cytoskeleton, PKA, A-kinase anchoring protein, Actin, Centrosome

\section{Introduction}

Protein phosphorylation is a tightly regulated process that is controlled by the opposing actions of protein kinases and phosphatases. It is now accepted that targeting of these signaling enzymes to distinct intracellular locations provides a mechanism to ensure specificity of signal transduction events by dictating which substrates are phosphorylated (Colledge and Scott, 1999). Accumulating evidence now demonstrates that pools of kinases and phosphatases are maintained in specific subcellular locations by anchoring and targeting proteins (Scott and Pawson, 2000).

The cyclic AMP signaling pathway has been the most extensively characterized signal transduction cascade (Sutherland, 1972). The second messenger cAMP mediates most of its cellular effects by activating the cAMP-dependent protein kinase (PKA). The PKA holoenzyme is a tetramer consisting of a regulatory subunit $(\mathrm{R})$ dimer and two catalytic subunits. To date, four genes encoding $\mathrm{R}$ subunits $(\mathrm{RI} \alpha, \mathrm{RI} \beta$, $\mathrm{RII} \alpha$ and $\mathrm{RII} \beta)$ and three genes encoding $\mathrm{C}$ subunits $(\mathrm{C} \alpha, \mathrm{C} \beta$ and $C \gamma$ ) have been identified (Taylor et al., 1990). Key cellular processes such as metabolism, gene transcription, ion channel conductivity, cell growth, cell division and actin cytoskeleton rearrangements are modulated by PKA-catalyzed phosphorylation in response to hormonal stimuli (Francis and Corbin, 1994; Scott, 1991). Given the broad spectrum of signaling events controlled by this kinase, it is crucial to understand the biochemical mechanisms that spatially and temporally regulate the PKA holoenzyme.

The intracellular organization of PKA is controlled through association with A-kinase-anchoring proteins (AKAPs). This family of functionally related proteins is classified on the basis of their ability to associate with the PKA holoenzyme inside cells (Colledge and Scott, 1999; Rubin, 1994). Each anchoring protein contains at least two functional motifs. The conserved PKA-binding motif forms an amphipathic helix of 14-18 residues that interacts with hydrophobic determinants located in the extreme N-terminus of the regulatory subunit dimer (Carr et al., 1991; Newlon et al., 1997; Newlon et al.,
1999; Fig. 1). Although most studies have focused on the interaction between AKAPs and RII, recent evidence indicates that AKAPs including D-AKAP1 (Huang et al., 1997b), D-AKAP2 (Huang et al., 1997a), AKAP82 (FCS1; Miki and Eddy, 1998), a Caenorhabditis elegans AKAP (AKAPce; Angelo and Rubin, 1998) and myosin VIIA (Kussel-Andermann et al., 2000) might also associate with RI. However, with the exception of AKAPce and possibly myosin VIIA, these anchoring proteins exhibit 10-20-fold higher affinities for RII over RI. In addition to the conserved $\mathrm{R}$ subunit binding surface, each AKAP contains a unique targeting motif that directs the PKA-AKAP complex to defined subcellular compartments (Colledge and Scott, 1999; Edwards and Scott, 2000).

Clearly the most significant property of several AKAPs is their ability to form multivalent signal transduction complexes (Fig. 1). By simultaneously interacting with multiple signaling enzymes, such as kinases or phosphatases, AKAPs can integrate diverse signaling pathways that coordinately regulate the phosphorylation of specific cellular substrates (Colledge and Scott, 1999). Accumulating evidence suggests that AKAPmediated organization of kinases and phosphatases is particularly important for the transduction of signals to the cytoskeleton. Here, we focus on the role of AKAPs in the targeting of signal transduction complexes to discrete sites of the cell cytoskeleton, including the centrosome, microtubules and actin.

\section{Targeting to the centrosome}

The centrosome is the major microtubule-nucleating organelle in animal cells and is usually composed of a pair of centrioles surrounded by a protein matrix from which microtubules are nucleated (Doxsey, 1998). Centrosomes ensure the fidelity of chromosome segregation during mitosis by guiding microtubule nucleation towards the formation of a bipolar spindle. During the past few years, an increasing number of kinases and phosphatases have been shown to be 


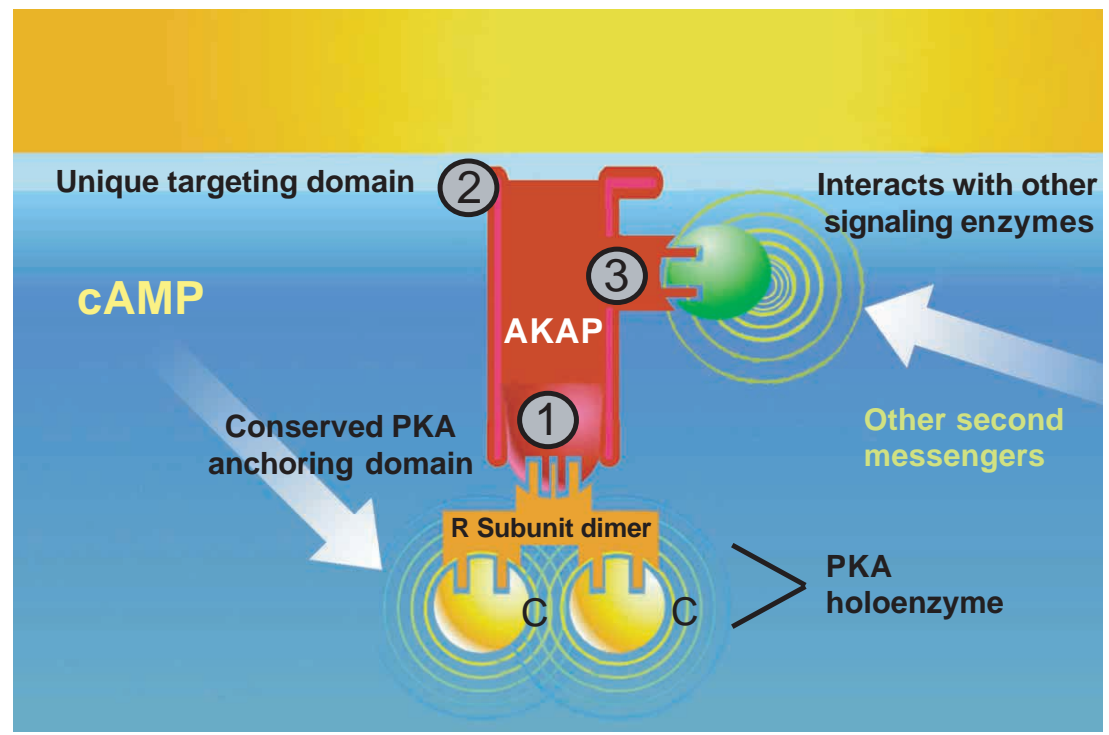

Fig. 1. Schematic representation of a prototypic AKAP signaling complex. The model illustrates the functional motifs of AKAPs. (1) A conserved RIIbinding domain interacts with the AKAP-binding surface on the regulatory subunit dimer of the PKA holoenzyme. (2) A unique targeting domain directs the AKAP signaling complex to discrete subcellular locations. (3) Docking sites for additional signaling enzymes, such as kinases and phosphatases, are also present. that a second distinct centrosomal protein, pericentrin, is also an AKAP (Diviani et al., 2000).

\section{AKAP350/AKAP450/CG-NAP}

Keryer et al. initially identified an AKAP in purified centrosomal fraction that had an apparent molecular mass of $350 \mathrm{kDa}$ (Keryer et al., 1993). Cloning and biochemical characterization of this molecule revealed that it has binding domains that interact with PKA, a binding site for the kinase PKN, which is activated by Rho and unsaturated fatty acids
Fig. 2. AKAPs at the centrosome and microtubules. (A) Schematic diagram depicting targeting of signaling enzymes to the centrosome and microtubules through anchoring proteins.

(B) Subcellular localization of centrosome- and microtubule-associated AKAPs. In each panel, the location of the AKAP is visualized by immunofluorescent labeling using antibodies specific for the indicated AKAP. In all cases, the green label indicates the AKAP, the red label indicates tubulin and the blue label indicates DNA.

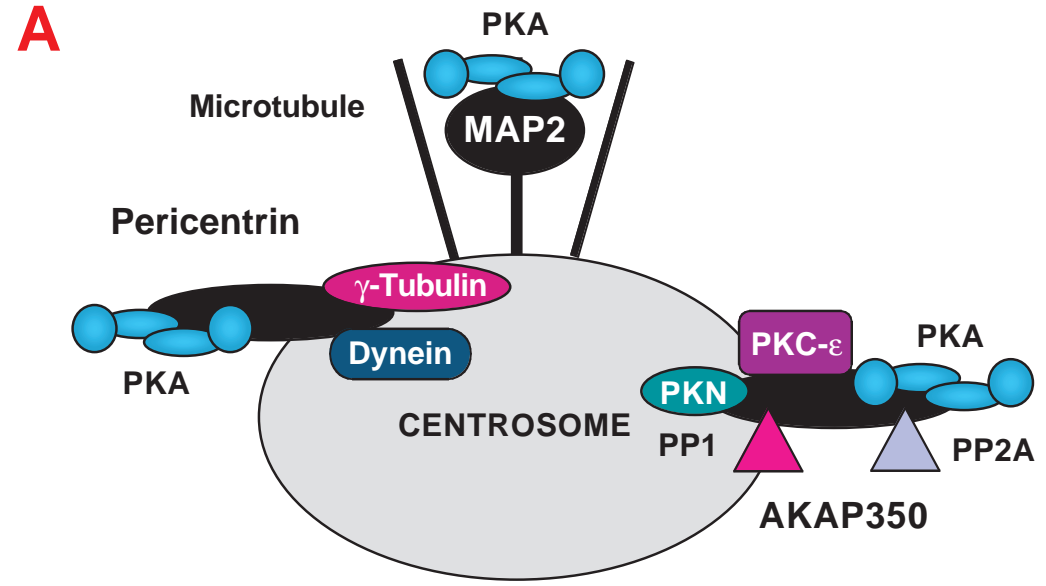

B
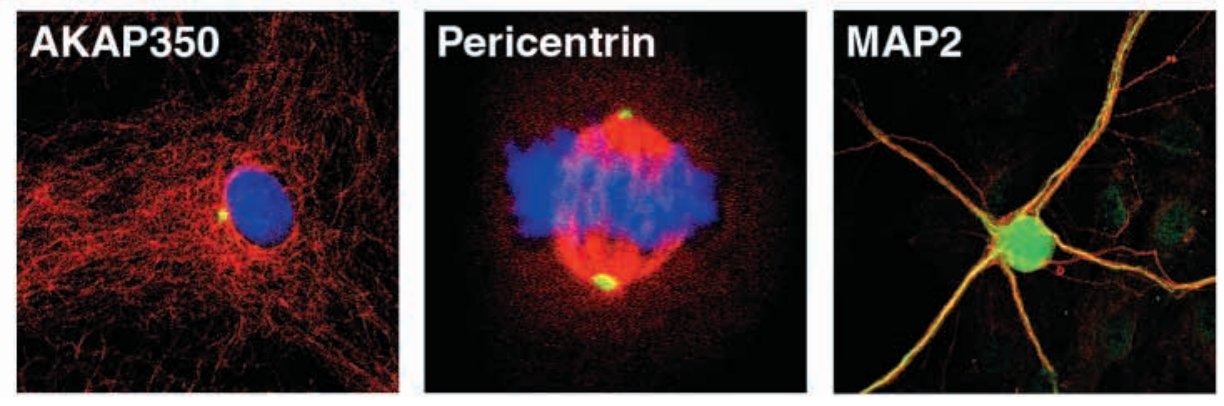
such as arachidonic acid, and docking sites for the phosphatases PP1 and PP2A (Takahashi et al., 1999; Fig. 2). Interestingly, AKAP350 contains two distinct RII-binding domains, which could potentially recruit two PKA holoenzymes per scaffolding unit. The type 1 phosphatase interacts through a conventional R/KVXF motif, a recognizable characteristic of many PP1-targeting subunits (Egloff et al., 1997), whereas tethering of the PP2A heterotrimer is mediated by the B subunit PR130 (Takahashi et al., 1999). More recent studies have shown that CG-NAP interacts with the immature non-phosphorylated form of PKCE (Takahashi et al., 2000; Fig. 2). This targeting interaction is required for the maturation of $\mathrm{PKC \varepsilon}$, which occurs upon phosphorylation at several sites on the enzyme. Collectively, these findings suggest that AKAP350 provides a point for the integration of distinct signaling pathways, including those involving cAMP, Rho, fatty acids and phospholipids.

AKAP350 expression is not restricted to the centrosomes: the protein is also found in the Golgi area and the cleavage furrow during distinct stages of the cell cycle (Schmidt et al., 1999). Thus, this anchoring protein might temporally control PKA location to permit access to compartment-specific PKA substrates at precise moments during cell division. The biochemical basis for docking of AKAP350 at the centrosome or to the Golgi area is yet to be elucidated. The gene encoding AKAP350 is found on chromosome 7q21. Sequence analysis has identified multiple exons that are alternatively spliced to produce at least four different isoforms. The isoforms are thought to exhibit different tissue expression patterns and are differentially compartmentalized (Schmidt et al., 1999). For example, the Yotiao protein, which is generated by alternative splicing of the $5^{\prime}$ end of the gene, is enriched in neurons and at the neuromuscular junction. This protein binds directly to the NR1A subtype of the NMDA-responsive glutamate receptor in neurons, in which a phosphatase-kinase signaling complex modulates the phosphorylation state and activity of the ion channel (Westphal et al., 1999). In contrast, AKAP350 is present in the centrosomes and Golgi in a variety of cell-types (Schmidt et al., 1999; Takahashi et al., 1999; Witczak et al., 1999). These data raise the intriguing possibility that different AKAP350 isoforms nucleate distinct combinations of signaling molecules at specific subcellular locations.

\section{Pericentrin}

Pericentrin is a highly conserved component of the centrosomal matrix originally identified as an antigen recognized by autoimmune sera from patients with scleroderma (Doxsey et al., 1994). This integral centrosomal protein provides a structural scaffold necessary for proper centrosomal architecture and microtubule nucleation during mitosis and meiosis (Dictenberg et al., 1998; Doxsey et al., 1994). Pericentrin interacts with $\gamma$-tubulin (Dictenberg et al., 1998) and the motor protein dynein (Purohit et al., 1999; Fig. $2 \mathrm{~A})$. Binding to $\gamma$-tubulin is required for the regulation of microtubule nucleation, whereas the association with dynein is necessary for the transport of pericentrin- $\gamma$-tubulin complexes along microtubules to the centrosome (Purohit et al., 1999; Young et al., 2000). Deletion of the dynein-binding domain results in the mistargeting of pericentrin and the generation of severe centrosomal defects (Purohit et al., 1999).

Pericentrin can also anchor the PKA holoenzyme (Diviani et al., 2000; Fig. 2). PKA anchoring is mediated through a 100residue, hydrophobic, binding domain that does not exhibit the structural characteristics of RII-binding sites on conventional AKAPs (Diviani et al., 2000). Leucine repeat sequences located in this region are likely to form an interactive surface with the canonical AKAP-binding determinant located in the $\mathrm{N}$-terminal region of the RII molecule. Recent results obtained in our laboratory show that disruption of PKA anchoring inside cells induces spindle abnormalities (D. Diviani, L. Langeberg, A. Purohit, A Young, S. Doxsey, and J. D. Scott, unpublished observations). Since dynein is a substrate for PKA (Inaba et al., 1998) and impairment of dynein function results in similar spindle defects, including spindle fragmentation and disorganization (Purohit et al., 1999), it is tempting to speculate that PKA anchored at the centrosome through association with pericentrin is involved in the regulation of dynein function.

The presence of two different AKAPs at the centrosome raises the intriguing possibility that, within a single cellular compartment, distinct anchoring proteins might target PKA, as well as other signaling enzymes, to precise sites to allow the phosphorylation of specific substrates. Thus AKAPs might not only direct the targeting of the PKA holoenzyme to a particular substrate but also spatially segregate independent signaling events within the same cellular compartment.

\section{Targeting to microtubules MAP2}

The microtubule-associated protein 2 (MAP2) family of proteins is an abundant group of cytoskeletal components that are predominantly expressed in neurons (Fig. 2b). These proteins participate in the stabilization of microtubules, the regulation of organelle transport within axons and dendrites, and the anchoring of signaling enzymes (Sanchez et al., 2000). MAP2 was the first protein shown to copurify and interact directly with the regulatory subunit of PKA (Theurkauf and Vallee, 1982; Fig. 2). Attachment to microtubules occurs through its tubulin-binding domain, which binds to an acidic region in the C-terminus of tubulin (Hirokawa, 1994; Serrano et al., 1984). Interestingly, this interaction might be regulated by PKA, because KXGS motifs located within the tubulin-binding domain of MAP2 are highly phosphorylated by the kinase (Hirokawa, 1994; Serrano et al., 1984). After phosphorylation, MAP2 exhibits decreased binding to tubulin, an increased rate of dissociation from microtubules and a reduction in microtubule-nucleating activity (Itoh et al., 1997). MAP2 is also phosphorylated by several other kinases including PKC, the $\mathrm{Ca}^{2+} /$ calmodulin-dependent kinase II (CAMKII), extracellularsignal-regulated kinases (ERKs), cyclin-dependent kinases (CDKs) and proline-directed protein kinases (PDPKs; Sanchez et al., 2000). Phosphorylation by these kinases also affects the binding affinity of MAP2 for tubulin. However, unlike PKA, none of these protein kinases has been shown to interact directly with MAP2.

Tau, another microtubule-binding protein structurally related to MAP2, directly interacts with PP2A (Sontag et al., 1996). This interaction is required for Tau dephosphorylation in vivo. The binding site for PP2A is conserved in MAP2; however, direct binding of the phosphatase has yet to be reported. No doubt, future studies will address whether MAP2 can act as a multivalent anchoring protein that coordinates the action of kinases and phosphatases at microtubules. 

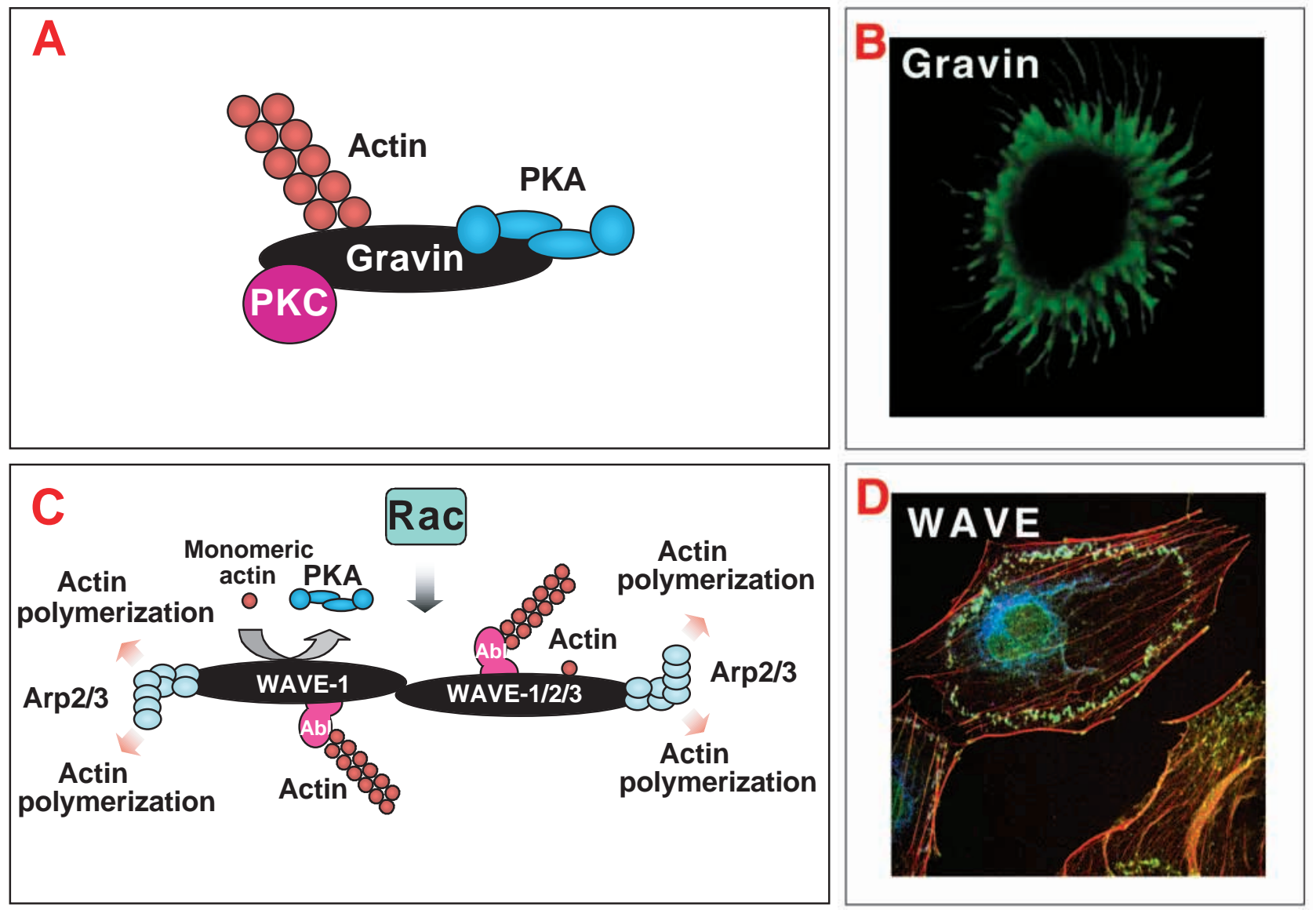

Fig. 3. AKAP at the actin cytoskeleton. (A) Schematic diagram depicting the gravin signaling complex. (B) Subcellular localization of gravin in human erythroleukemia (HEL) cells. Gravin expression was visualized by immunofluorescent labeling using gravin-specific antibodies after treatment of HEL cells for $18 \mathrm{~h}$ with phorbol esters. (C) Model illustrating the WAVE signaling complex crosslinked to the actin cytoskeleton. Components of the PKA holoenzyme, the Arp2/3 complex and F actin are indicated. Individual proteins associated with a WAVE-1-WAVE2/3 dimer are labeled. (D) Subcellular localization of the WAVE1 signaling complex in Swiss 3T3 fibroblasts. Immunohistochemical analysis used antibodies against WAVE1 (green) and RII (blue). Actin (red) was detected by Texas-red-phalloidin.

\section{Targeting to the actin cytoskeleton}

The actin cytoskeleton is critical for a variety of essential biological processes in all eukaryotic cells, including the establishment of cell shape and polarity, motility, and cell division (Hall, 1998). A fundamental question is how cells integrate signals from a variety of pathways to control the precise location and the timing of actin polymerization. Key players are members of the Rho family of small GTPases, which have emerged as the principal transmitters of signals from transmembrane receptors that stimulate actin filament nucleation (Bishop and Hall, 2000). The most extensively characterized members are Rho, Rac and Cdc42, which control the formation of actin stress fibers, lamellipodia and filopodia, respectively. These distinct actinremodeling events are a consequence of the selective interaction of the activated Rho GTPases with specific effector proteins. Recent evidence indicates that actinbinding proteins such as gravin (an antigen for the autoimmune disease myasthenia gravis) and WAVE (a member of the Wiskott-Aldrich syndrome protein (WASP) family of adapter proteins) are AKAPs (Nauert et al., 1997; Westphal et al., 2000).

\section{Gravin}

Gravin originally was identified as a cytoplasmic antigen recognized by sera from patients suffering from myasthenia gravis, an autoimmune degenerative disease that primarily affects transmission at the neuromuscular junctions (Gordon et al., 1992). The cloning and biochemical characterization of gravin revealed that it is a multivalent $250-\mathrm{kDa}$ kinase-scaffold protein that interacts with PKA, PKC and actin (Gordon et al., 1992; Nauert et al., 1997; Fig. 3A). Gravin expression is induced by phorbol esters in variety of endothelial, macrophage-like cells, in which the protein localizes to filopodia (Fig. 3B). Gravin shares significant homology with SSeCKS (also called clone 72), a cell-cycle-regulated myristylated PKC substrate that has tumor suppressor activity (Frankfort and Gelman, 1995; Lin et al., 1996; Nelson and Gelman, 1997). SSeCKs, which binds to PKA, PKC and the actin cytoskeleton (Erlichman et al., 1999), mediates distinct actin-remodeling events (Gelman et al., 1998). In fact, ectopic expression of SSeCKS in NIH-3T3 fibroblasts causes significant cell flattening, the loss of actin stress fibers and the elaboration of SSeCKS-associated filopodia-like projections. In addition, SSeCKS is implicated in control of cell migration 
during mouse embryogenesis (Gelman et al., 1998). Recently, Shih et al. showed that a gravin-PKA-PKC signaling complex interacts with the $\beta 2$ adrenergic receptor in human epidermoid carcinoma cells (Shih et al., 1999). Inhibition of gravin expression disrupts the recycling of this receptor after agonistinduced desensitization (Shih et al., 1999). Since PKC is directly involved in $\beta 2$ adrenergic receptor resensitization (Shih et al., 1999), it is tempting to speculate that gravin modulates recycling by directing $\mathrm{PKC}$ in proximity of the receptor.

In conclusion, gravin has been shown to play very diverse functions that range from the modulation of cytotoskeletal rearrangements to the regulation of G-protein-coupled receptors. No doubt future studies will address whether these functions are regulated by the signaling enzymes associated with gravin.

\section{The Wiskott-Aldrich syndrome protein (WASP) family}

The WASP family of proteins has five known members: WASP, N-WASP, Scar-1 and three WAVE isoforms (Takenawa and Miki, 2001). WASP, the founding member of the family, is mutated in Wiskott-Aldrich syndrome, an X-linked human immunodeficiency disease (Snapper and Rosen, 1999). The WASP homologue N-WASP is expressed ubiquitously in vertebrate cells and causes the formation of filopodia when coexpressed with Cdc42 (Miki et al., 1998a). Scar-1 was discovered in Dictyostelium in a genetic screen for proteins downstream of the chemotaxis receptor for cyclic AMP, cAR2 (Bear et al., 1998). More recently, three mammalian orthologues of Scar-1, termed WAVE1, WAVE2 and WAVE3, which are involved in Rac1-induced actin reorganization, have been cloned (Miki et al., 1998b; Suetsugu et al., 1999; Fig. 3C). WASP family members provide a molecular bridge that functionally couples individual Rho GTPases to the Arp2/3 complex, a group of seven related proteins that can nucleate actin polymerization (Higgs and Pollard, 1999; Machesky and Gould, 1999). The N-terminal regions of WASP and N-WASP contain a CRIB domain that mediates the direct interaction with the activated form of Cdc42; the three WAVE isoforms are coupled with Rac through an as-yet-undefined mechanism (Kim et al., 2000; Mullins, 2000). The central portion of each WASP family member contains proline-rich sequences that interact with $\mathrm{SH} 3$ proteins, the G-actin-binding protein profilin and other actin-associated proteins (Miki et al., 1998b; Mullins, 2000). Direct attachment to the actin cytoskeleton occurs through two conserved binding motifs: a verprolin homology (VPH) domain and a C-terminal acidic module that binds to the Arp2/3 complex (Machesky and Gould, 1999; Fig. 3C).

Recently, WAVE1 was shown to bind to both PKA and the Abl tyrosine kinase (Westphal et al., 2000; Fig. 3C). Abl binding appears to be a common characteristic of the WAVE family, because WAVE2 and WAVE3 also interact with the Abl SH3 domain (R. S. Westphal and J. D. Scott, unpublished). Only the WAVE1 isoform, however, binds to PKA. Interestingly, the RII-binding region of WAVE1 overlaps a VPH domain previously characterized as a binding site for Gactin. In vitro competition experiments show that actin competes for the RII-binding site (Westphal et al., 2000; Fig. 3C). This might provide a mechanism for the regulation of PKA anchoring at sites of actin reorganization, at which the local actin concentration might be sufficient to displace the anchored PKA. Another interesting property of the WAVE isoforms is their ability to homo- and hetero-dimerize. This provides an additional level of organization, since signaling units containing various WAVE isoforms might be nucleated at distinct sites of actin reorganization (Westphal et al., 2000). Finally, assembly of the WAVE1 signaling complex is dependent upon extracellular stimuli. Activation of Rac by PDGF results in a rapid redistribution of WAVE1, PKA and Abl to lamellipodia and actin ring structures at the periphery of the cell (Westphal et al., 2000; Fig. 3D). Dynamic assembly of WAVE signaling complexes could be a sophisticated mechanism to coordinate the location and action of PKA and $\mathrm{Abl}$ in response to extracellular signals.

\section{Other cytoskeleton-associated AKAPs AKAP-KL}

AKAP-KL is a cytoskeleton-associated anchoring protein that is almost exclusively expressed in kidney, lung and cerebellum (Dong et al., 1998). In polarized lung and kidney epithelial cells, AKAP-KL is selectively targeted to the inner, apical surface. This particular intracellular distribution is maintained through a tight association with the cortical actin cytoskeleton. It is currently unknown whether AKAP-KL binds directly to actin or interacts with actin-associated proteins. The gene encoding AKAP-KL is alternatively spliced to generate at least six different isoforms (Dong et al., 1998). Targeting of AKAP-KL-PKA signaling complexes near the junction of cytoskeleton and apical membrane might facilitate cyclicAMP-mediated trans-epithelial signal transduction (Dong et al., 1998).

\section{AKAP82 and AKAP110}

AKAP82 (Carrera et al., 1994; Miki and Eddy, 1998) and AKAP110 (Vijayaraghavan et al., 1999) are two cytoskeletal anchoring proteins found in male germ cells. In vitro studies suggest that AKAP82 (also called FSC1) is a dual-specificity anchoring protein that contains tethering domains for both RII $\alpha$ and RI $\alpha$. AKAP82 is the major structural protein of the fibrous sheath in the principal piece of the flagellum, a unique cytoskeletal structure believed to modulate sperm motility, whereas AKAP110 expression is mostly localized in the acrosomal region of the head and along the entire length of the flagellum (Vijayaraghavan et al., 1999). Sperm motility is regulated by PKA-mediated phosphorylation of a group of largely unidentified flagellar proteins (Miki and Eddy, 1998). By tethering PKA to distinct cytoskeletal structures of the flagellum of human spermatozoa, AKAP82 and AKAP110 might regulate the phosphorylation state of specific protein targets and therefore control sperm motility.

\section{Conclusions and perspectives}

Over the past decade, AKAPs have emerged as key regulators of PKA function. By tethering the holoenzyme at precise cell locations, in close proximity to their physiological substrate(s), AKAPs favor specific PKA phosphorylation events. It is now apparent that several AKAPs are targeted to the same subcellular compartment (Colledge and Scott, 1999; Edwards and Scott, 2000). This is the case for pericentrin and 
AKAP350, which both target PKA to the centrosome. This apparent redundancy in PKA anchoring might reflect the exquisite level of compartmentalization mediated by different AKAP signaling complexes. In essence, a handful of substrate molecules may be serviced by an individual AKAP signaling complex. Future efforts will focus on delineating substrates that are regulated by individual AKAP transduction units. Different approaches might address this question. First, soluble inhibitors of PKA anchoring that could selectively disrupt the interaction of a specified AKAP with PKA could be generated. Alternatively, a more physiological approach that will address the relevance of PKA anchoring in vivo would be the development of transgenic and knockout mice. PKA function has already been investigated in recent elegant studies that have systematically ablated the genes encoding $\mathrm{R}$ and $\mathrm{C}$ subunits of the PKA holoenzyme (Brandon et al., 1997). No doubt, genetic experiments on AKAPs will complement these studies and greatly extend our current knowledge of the physiological importance of AKAP-based signal transduction complexes.

The interaction between AKAP and RII has been extensively characterized, and the NMR structures of different AKAP-RII complexes have now been solved (Newlon et al., 1997; Newlon et al., 1999). Because of the high affinity of the RII-AKAP complex, this interaction was considered to be constitutive in cells. However, recent data clearly demonstrate that actin and RII compete for binding to Wave1 (Westphal et al., 2000). This represents the first example of a dynamically regulated AKAPPKA interaction. Another elegant example of dynamic regulation is suggested by indirect experimental evidence showing that the association of RII $\alpha$ with the centrosome is regulated during the cell cycle (Keryer et al., 1998). Future studies will undoubtedly identify centrosomal AKAPs (pericentrin or AKAP350) involved in this regulated interaction.

AKAPs clearly maintain signaling complexes through the interaction with various signaling enzymes (Colledge and Scott, 1999). Recent examples are AKAP350, which binds to PKA, PKN, PKC- $\varepsilon$, PP1 and PP2A (Takahashi et al., 2000; Takahashi et al., 1999), and Wave1, which forms complexes with PKA, Abl and Arp2/3 at sites of actin reorganization (Westphal et al., 2000). This property is of particular interest because it provides the molecular basis for the integration of different signaling pathways within a single transduction unit. Now that many of the AKAP interactions have been identified, a major task will be to understand the dynamics of signaling complex assembly and to identify kinase substrates recruited into these signal transduction units.

We thank the colleagues in the Scott lab for critically evaluating the manuscript and Lorene Langeberg for providing the confocal images. Supported in part by DK44239 to J. D. S.

\section{References}

Angelo, R. and Rubin, C. S. (1998). Molecular characterization of an anchor protein $\left(\mathrm{AKAP} \mathrm{CE}_{\mathrm{E}}\right)$ that binds the RI subunit $\left(\mathrm{R}_{\mathrm{CE}}\right)$ of type I protein kinase A from Caenorhabditis elegans. J. Biol. Chem. 273, 14633-14643.

Bear, J. E., Rawls, J. F. and Saxe, C. L. III (1998). SCAR, a WASP-related protein, isolated as a suppressor of receptor defects in late Dictyostelium development. J. Cell Biol. 142, 1325-1335.

Bischoff, J. R., Anderson, L., Zhu, Y., Mossie, K., Ng, L., Souza, B., Schryver, B., Flanagan, P., Clairvoyant, F., Ginther, C. et al. (1998). A homologue of Drosophila aurora kinase is oncogenic and amplified in human colorectal cancers. EMBO J. 17, 3052-3065.

Bishop, A. L. and Hall, A. (2000). Rho GTPases and their effector proteins. Biochem J. 348, 241-255.

Brandon, E. P., Idzerda, R. L. and McKnight, G. S. (1997). PKA isoforms, neural pathways, and behaviour: making the connection. Curr. Opin. Neurobiol. 7, 397-403.

Carr, D. W., Stofko-Hahn, R. E., Fraser, I. D. C., Bishop, S. M., Acott, T. S., Brennan, R. G. and Scott, J. D. (1991). Interaction of the regulatory subunit (RII) of cAMP-dependent protein kinase with RII-anchoring proteins occurs through an amphipathic helix binding motif. J. Biol. Chem. 266, 14188-14192.

Carrera, A., Gerton, G. L. and Moss, S. B. (1994). The major fibrous sheath polypeptide of mouse sperm: structural and functional similarities to the Akinase anchoring proteins. Dev. Biol. 165, 272-284.

Colledge, M. and Scott, J. D. (1999). AKAPs: from structure to function. Trends Cell Biol. 9, 216-221.

De Camilli, P., Moretti, M., Donini, S. D., Walter, U. and Lohmann, S. M. (1986). Heterogenous distribution of the cAMP receptor protein RII in the nervous system: evidence for its intracellular accumulation on microtubules, microtubule-organizing centers, and in the area of the Golgi complex. J. Cell Biol. 103, 189-203.

Dictenberg, J. B., Zimmerman, W., Sparks, C. A., Young, A., Vidair, C., Zheng, Y., Carrington, W., Fay, F. S. and Doxsey, S. J. (1998). Pericentrin and gamma-tubulin form a protein complex and are organized into a novel lattice at the centrosome. J. Cell Biol. 141, 163-174.

Diviani, D., Langeberg, L. K., Doxsey, S. J. and Scott, J. D. (2000). Pericentrin anchors protein kinase A at the centrosome through a newly identified RII-binding domain. Curr. Biol. 10, 417-420.

Dong, F., Felsmesser, M., Casadevall, A. and Rubin, C. S. (1998). Molecular characterization of a cDNA that encodes six isoforms of a novel murine A kinase anchor protein. J. Biol. Chem. 273, 6533-6541.

Doxsey, S. J., Stein, P., Evans, L., Calarco, P. D. and Kirschner, M. (1994). Pericentrin, a highly conserved centrosome protein involved in microtubule organization. Cell 76, 639-650.

Doxsey, S. (1998). The centrosome - a tiny organelle with big potential [news; comment]. Nature Genet. 20, 104-106.

Edwards, A. S. and Scott, J. D. (2000). A-kinase anchoring proteins: protein kinase A and beyond. Curr. Opin. Cell Biol. 12, 217-221.

Egloff, M. P., Johnson, D. F., Moorhead, G., Cohen, P. T. W., Cohen, P. and Barford, D. (1997). Structural basis for the recognition of regulatory subunits by the catalytic subunit of protein phosphatase 1. EMBO J. 16, 1876-1887.

Erlichman, J., Gutierrez-Juarez, R., Zucker, S., Mei, X. and Orr, G. A. (1999). Developmental expression of the protein kinase C substrate/binding protein (clone $72 / \mathrm{SSeCKS}$ ) in rat testis identification as a scaffolding protein containing an A-kinase-anchoring domain which is expressed during latestage spermatogenesis. Eur. J. Biochem. 263, 797-805.

Francis, S. H. and Corbin, J. D. (1994). Structure and function of cyclic nuleotide-dependent protein kinases. Annu. Rev. Physiol. 56, 237-272.

Frankfort, B. J. and Gelman, I. H. (1995). Identification of novel cellular genes transcriptionally suppressed by v-src. Biochem. Biophys. Res. Commun. 206, 916-926.

Gelman, I. H., Lee, K., Tombler, E., Gordon, R. and Lin, X. (1998). Control of cytoskeletal architecture by the src-suppressed C kinase substrate, SSeCKS. Cell Motil. Cytoskel. 41, 1-17.

Gordon, T., Grove, B., Loftus, J. C., O'Toole, T., McMillan, R., Lindstrom, J. and Ginsberg, M. H. (1992). Molecular cloning and preliminary characterization of a novel cytoplasmic antigen recognized by myasthenia gravis sera. J. Clin. Invest. 90, 992-999.

Hall, A. (1998). Rho GTPases and the actin cytoskeleton. Science 279, 509514.

Higgs, H. N. and Pollard, T. D. (1999). Regulation of actin polymerization by Arp2/3 complex and WASp/Scar proteins. J. Biol. Chem. 274, 3253132534.

Hinchcliffe, E. H., Li, C., Thompson, E. A., Maller, J. L. and Sluder, G. (1999). Requirement of Cdk2-cyclin E activity for repeated centrosome reproduction in Xenopus egg extracts. Science 283, 851-854.

Hirokawa, N. (1994). Microtubule organization and dynamics dependent on microtubule-associated proteins. Curr. Opin. Cell Biol. 6, 74-81.

Huang, L. J., Durick, K., Weiner, J. A., Chun, J. and Taylor, S. S. (1997a). D-AKAP2, a novel protein kinase A anchoring protein with a putative RGS domain. Proc. Nat. Acad. Sci. USA 94, 11184-11189.

Huang, L. J., Durick, K., Weiner, J. A., Chun, J. and Taylor, S. S. (1997b). 
Identification of a novel dual specificity protein kinase A anchoring protein, D-AKAP1. J. Biol. Chem. 272, 8057-8064.

Inaba, K., Morisawa, S. and Morisawa, M. (1998). Proteasomes regulate the motility of salmonid fish sperm through modulation of cAMP-dependent phosphorylation of an outer arm dynein light chain. J. Cell Sci. 111, 11051115 .

Itoh, T. J., Hisanaga, S., Hosoi, T., Kishimoto, T. and Hotani, H. (1997). Phosphorylation states of microtubule-associated protein 2 (MAP2) determine the regulatory role of MAP2 in microtubule dynamics. Biochemistry 36, 12574-12582.

Keryer, G., Rios, R. M., Landmark, B. F., Skalhegg, B., Lohmann, S. M. and Bornens, M. (1993). A high-affinity binding protein for the regulatory subunit of cAMP-dependent protein kinase II in the centrosome of human cells. Exp. Cell Res. 204, 230-240.

Keryer, G., Yassenko, M., Labbe, J. C., Castro, A., Lohmann, S. M., EvainBrion, D. and Tasken, K. (1998). Mitosis-specific phosphorylation and subcellular redistribution of the RIIalpha regulatory subunit of cAMPdependent protein kinase. J. Biol. Chem. 273, 34594-34602.

Kim, A. S., Kakalis, L. T., Abdul-Manan, N., Liu, G. A. and Rosen, M. K. (2000). Autoinhibition and activation mechanisms of the Wiskott-Aldrich syndrome protein. Nature 404, 151-158.

Kussel-Andermann, P., El-Amraoui, A., Safieddine, S., Hardelin, J. P., Nouaille, S., Camonis, J. and Petit, C. (2000). Unconventional myosin VIIA is a novel A-kinase-anchoring protein. J. Biol. Chem. 275, 2965429659.

Lin, X., Tombler, E., Nelson, P. J., Ross, M. and Gelman, I. H. (1996). A novel src- and ras-suppressed protein kinase C substrate associated with cytoskeletal architecture. J. Biol. Chem. 271, 28430-28438.

Machesky, L. M. and Gould, K. L. (1999). The Arp2/3 complex: a multifunctional actin organizer. Curr. Opin. Cell Biol. 11, 117-121.

Miki, K. and Eddy, E. M. (1998). Identification of tethering domains for protein kinase A type Ialpha regulatory subunits on sperm fibrous sheath protein FSC1. J. Biol. Chem. 273, 34384-34390.

Miki, H., Sasaki, T., Takai, Y. and Takenawa, T. (1998a). Induction of filopodium formation by a WASP-related actin-depolymerizing protein NWASP. Nature 391, 93-96.

Miki, H., Suetsugu, S. and Takenawa, T. (1998b). WAVE, a novel WASPfamily protein involved in actin reorganization induced by Rac. EMBO J. 17, 6932-6941.

Mullins, R. D. (2000). How WASP-family proteins and the Arp2/3 complex convert intracellular signals into cytoskeletal structures. Curr. Opin. Cell Biol. 12, 91-96.

Nauert, J. B., Klauck, T. M., Langeberg, L. K. and Scott, J. D. (1997). Gravin, an autoantigen recognized by serum from myasthenia gravis patients, is a kinase scaffold protein. Curr. Biol. 7, 52-62.

Nelson, P. J. and Gelman, I. H. (1997). Cell-cycle regulated expression and serine phosphorylation of the myristylated protein kinase $\mathrm{C}$ substrate, SSeCKS: correlation with culture confluency, cell cycle phase and serum response. Mol. Cell. Biochem. 175, 233-241.

Newlon, M. G., Roy, M., Hausken, Z. E., Scott, J. D. and Jennings, P. A. (1997). The A-kinase anchoring domain of Type II $\alpha$ cAMP-dependent protein kinase is highly helical. J. Biol. Chem. 272, $23637-23644$.

Newlon, M. G., Roy, M., Morikis, D., Hausken, Z. E., Coghlan, V., Scott, J. D. and Jennings, P. A. (1999). The molecular basis for protein kinase A anchoring revealed by solution NMR. Nature Struct. Biol. 6, 222-227.

Nigg, E. A. (1998). Polo-like kinases: positive regulators of cell division from start to finish. Curr. Opin. Cell Biol. 10, 776-783.

Nigg, E. A., Schafer, G., Hilz, H. and Eppenberger, H. M. (1985). CyclicAMP-dependent protein kinase type II is associated with the Golgi complex and with centrosomes. Cell 41, 1039-1051.

Purohit, A., Tynan, S. H., Vallee, R. and Doxsey, S. J. (1999). Direct interaction of pericentrin with cytoplasmic dynein light intermediate chain contributes to mitotic spindle organization. J. Cell Biol. 147, 481-492.

Rubin, C. S. (1994). A kinase anchor proteins and the intracellular targeting of signals carried by cAMP. Biochim. Biophys. Acta 1224, 467-479.

Sanchez, C., Diaz-Nido, J. and Avila, J. (2000). Phosphorylation of microtubule-associated protein 2 (MAP2) and its relevance for the regulation of the neuronal cytoskeleton function. Prog. Neurobiol. 61, 133168.

Schmidt, P. H., Dransfield, D. T., Claudio, J. O., Hawley, R. G., Trotter, K. W., Milgram, S. L. and Goldenring, J. R. (1999). AKAP350: a multiply spliced A-kinase anchoring protein associated with centrosomes. J. Biol. Chem. 274, 3055-3066.

Scott, J. D. (1991). Cyclic nucleotide-dependent protein kinases. Pharmacol. Ther. 50, 123-145.

Scott, J. D. and Pawson, T. (2000). Cell communication: the inside story. Sci. Am. 282, 72-79.

Serrano, L., Avila, J. and Maccioni, R. B. (1984). Controlled proteolysis of tubulin by subtilisin: localization of the site for MAP2 interaction. Biochemistry 23, 4675-4681.

Shih, M., Lin, F., Scott, J. D., Wang, H. Y. and Malbon, C. C. (1999). Dynamic complexes of beta2-adrenergic receptors with protein kinases and phosphatases and the role of gravin. J. Biol. Chem. 274, 1588-1595.

Snapper, S. B. and Rosen, F. S. (1999). The Wiskott-Aldrich syndrome protein (WASP): roles in signaling and cytoskeletal organization. Annu. Rev. Immunol. 17, 905-929.

Sontag, E., Nunbhakdi-Craig, V., Lee, G., Bloom, G. S. and Mumby, M. C. (1996). Regulation of the phosphorylation state and microtubule-binding activity of tau by protein phosphatase 2A. Neuron 17, 1201-1207.

Suetsugu, S., Miki, H. and Takenawa, T. (1999). Identification of two human WAVE/SCAR homologues as general actin regulatory molecules which associate with the Arp2/3 complex. Biochem. Biophys. Res. Commun. 260, 296-302.

Sutherland, E. W. (1972). Studies on the mechanism of hormone action. Science 171, 401-408.

Takahashi, M., Shibata, H., Shimakawa, M., Miyamoto, M., Mukai, H. and Ono, Y. (1999). Characterization of a novel giant scaffolding protein, CG-NAP, that anchors multiple signaling enzymes to centrosome and the golgi apparatus. J. Biol. Chem. 274, 17267-17274.

Takahashi, M., Mukai, H., Oishi, K., Isagawa, T. and Ono, Y. (2000). Association of immature hypo-phosphorylated protein kinase $\mathrm{C}$ epsilon with an anchoring protein CG-NAP. J. Biol. Chem. 275, 34592-34596.

Takenawa, T. and Miki, H. (2001). WASP and WAVE family proteins, key molecules for rapid rearrangement of cortical actin filaments and cell movement. J. Cell Sci. (in press).

Taylor, S. S., Buechler, J. A. and Yonemoto, W. (1990). cAMP-dependent protein kinase: framework for a diverse family of regulatory enzymes. Annu. Rev. Biochem. 59, 971-1005.

Theurkauf, W. E. and Vallee, R. B. (1982). Molecular characterization of the cAMP-dependent protein kinase bound to microtubule-associated protein 2 . J. Biol. Chem. 257, 3284-3290.

Vijayaraghavan, S., Liberty, G. A., Mohan, J., Winfrey, V. P., Olson, G. E. and Carr, D. W. (1999). Isolation and molecular characterization of AKAP110, a novel, sperm- specific protein kinase A-anchoring protein. Mol. Endocrinol. 13, 705-717.

Westphal, R. S., Tavalin, S. J., Lin, J. W., Alto, N. M., Fraser, I. D., Langeberg, L. K., Sheng, M. and Scott, J. D. (1999). Regulation of NMDA receptors by an associated phosphatase-kinase signaling complex. Science 285, 93-96.

Westphal, R. S., Soderling, S. H., Alto, N. M., Langeberg, L. K. and Scott, J. D. (2000). Scar/WAVE-1, a wiskott-aldrich syndrome protein, assembles an actin-associated multi-kinase scaffold. EMBO J. 19, 4589-4600.

Witczak, O., Skalhegg, B. S., Keryer, G., Bornens, M., Tasken, K., Jahnsen, T. and Orstavik, S. (1999). Cloning and characterization of a cDNA encoding an A-kinase anchoring protein located in the centrosome, AKAP450. EMBO J. 18, 1858-1868.

Young, A., Dictenberg, J. B., Purohit, A., Tuft, R. and Doxsey, S. J. (2000). Cytoplasmic dynein-mediated assembly of pericentrin and gamma tubulin onto centrosomes. Mol. Biol. Cell 11, 2047-2056.

Zhou, H., Kuang, J., Zhong, L., Kuo, W. L., Gray, J. W., Sahin, A., Brinkley, B. R. and Sen, S. (1998). Tumour amplified kinase STK15/BTAK induces centrosome amplification, aneuploidy and transformation. Nature Genet. 20, 189-193. 